\title{
Innovations and development in the marketing supply chain: Migrating brand creation from passive to direct interaction with the consumer - Interview with Subhanker Bhattacharya of HCL Technologies
}

\begin{abstract}
Subhankar Bhattacharya
As a Principal with HCL's Media and Entertainment industry vertical, Subhankar Bhattacharya is responsible for shaping multiple "Go To" market solutions for HCL. At the moment, he is spearheading the SOA-based digital media supply chain framework "IMPACT", which will facilitate how companies optimize their investments in digital media and ensure faster go-to-market for new services. In the past couple of years, Subhankar has helped multiple companies in the media and publishing space design and implement digital media solutions for marketing, production and distribution automation. Subhankar is a regular speaker in premier conferences like NAB and ESCA. He has published multiple papers across internal and external publications on digital and physical media supply chains.
\end{abstract}

Keywords: marketing supply chain, web, mobile, broadcast, marketing measurement, service-oriented architecture

Abstract As marketing organizations begin to migrate value out of the traditional agency and service provider network and into their own operations, how has the web accelerated that migration of value? How has this changed the entertainment industry? We spoke with Subhankar Bhattacharya, Principal of Media and Entertainment at HCL America, on the impact of social networking on marketing operations - and a general look at the future of the marketing supply chain. Journal of Digital Asset Management (2008) 4, 188-196. doi:10.1057/dam.2008.31

Subhankar Bhattacharya Media \& Entertainment, HCL America,

18301 Von Karman Avenue Suite 590 , Irvine, CA-92612 USA

Tel: 9492325510 E-mail: sbhattacharya@ hcl.in
MM: Why don't we start off with a brief introduction? Then a little bit about your company.

SB: Sure. My name is Subhankar Bhattacharya. I am a principal with the Media and Entertainment Practice of HCL Technologies. I have 13 years of experience, eight years of which I've spent in the industry and five years on the consulting side.

Just to give you a little brief about my company - HCL Technologies is part of the $\$ 4.7 \mathrm{bn}$ HCL Enterprise. The company has been in operation for more than 30 years. As an enterprise, HCL had its genesis in R\&D. In fact, many people may not be aware that HCL introduced multiprocessor Unix in 1989, three years ahead of Sun and HP. Media and entertainment is one of the key verticals that HCL focuses on.

MM: What are some of the other verticals that HCL provides IT and related services to? SB: HCL has deep expertise servicing several verticals, including finance, retail, telecom, hi-tech, manufacturing, aerospace, automotive, healthcare and media and entertainment.

MM: Today we will explore what you see as some of the emerging trends and developments in what we call the marketing supply chain and related technologies that support marketing and innovation within the marketing operations.

What are some of the first things that come to mind in terms of innovations and development in the marketing supply chain? 
SB: One of the things that probably comes to mind immediately is that the marketing supply chain has come a long way from a completely analog or paper-based environment to a completely digital supply chain, within a span of a decade and half. That's a fairly quick pace of development.

For a long time we had pretty standard analog processes. You nail down a concept, prepare artwork, go through the discrete approval processes and follow up with production. It used to take a significantly long time from concept to launch. We had quite a settled down relationship among companies, ad agencies and production houses and nothing changed for a long time.

I think the change started happening in the early 90s. To begin with, ad agencies started maintaining the content in digital form for their own use. Slowly they started sharing the digital content with corporate. First it was offline through CDs and then moved online - the approval processes and production orders started moving online too. There was a lot of automation taking place.

However, many of these automations were driven from the ad agency point of view. What we've seen in the last five years or so is that the organizations themselves have started taking a lot of control in the ad content that they have. There is a clear trend toward marketing solutions going into complete end-to-end automated mode driven from within the organizations. This end-to-end automation encompasses everything from conception to distribution, and further extending to marketing effectiveness measurement. This is a pretty significant change in terms of control of the entire marketing process and all that has happened in the last 12-15 years.

MM: As marketing organizations have begun to migrate value out of the traditional agency and service provider network, and have migrated into their own operations, how has the web and mobile accelerated that migration of value? SB: Since the beginning of the dot com era, the importance of web marketing has grown significantly. What has made the most telling impact in this value creation, however, is the emergence of the social networking platforms. Before the emergence of these platforms, we were still talking about search ads and banner ads. Now the entire marketing game has changed. One successful viral campaign through web 2.0 platform may stand in between a successful movie launch and a failed one. Marketing always had this standard model about speed of information sharing. I think social networks have crushed all those barriers. Additionally, when we talk about mobile, it is once again not a stand-alone device any more, but it is all part of the expansive world wide web. So the impacts of social networks on mass marketing and the effects of mobile on the speed of communication have all been absolutely dramatic.

MM: We've also seen the expanse of the role of marketing analytics. Initially, driven by two groups: the traditional "market research/ customer research" group that did their focus groups and surveys and satisfaction assessments, as well as the faster moving and perhaps less sophisticated "web analytics" group.

We're now beginning to see the integration of the traditional insights and customer insights in analytics with the more dynamic, real-time multidimensional web analytics.

Could you speak to what you've seen in terms of the emergence of what we now call "multichannel marketing analytics?"

SB: Yes. Absolutely. I think that the point you're drawing here is so relevant today.

In a traditional marketing approach we had typical distribution channels for marketing. Everybody knew where they were putting their content, what kind of market they were reaching and what kind of response they could expect. Today we have new channels of marketing communication and most content also has a very short lifecycle (if we leave aside the long tail).

As a result, marketing today is so extremely dynamic. For example, you have a new movie launch, and you create a short game out of that movie theme and initiate viral marketing with that content.

Now most of this stuff is new and quite a lot is experimental. Just like you can't predict the success of a movie, this kind of marketing effectiveness is, however, hard to predict.

At the same time, if you do not have a complete grip on how your innovations in marketing are paying off, you'll probably be 
shooting in the dark, and spending money on initiatives that you really don't want.

If something is hard to forecast but you can't do without it, the first thing you do is create transparency in the whole process. That means, even if you are not forecasting, you must know in real-time how well it is working. This is probably very similar to retail space, where there is always a tug of war between investments in the forecasting engine vs supply chain visibility.

The situation in traditional marketing channels, such as TV, magazines, etc., is rather stable but these channels also are also affected by online growth. This is impacting the upfront ad market and hence reducing the overall predictability of the traditional channels.

Hence, when we talk about multichannel marketing analytics, I see more of transparency than prediction as the basic driving force. However, at the same time, marketing transparency also includes complex analytics such as real time market segmentation and decision support system based on such data. MM: Another thing we've seen that meshes with what you're saying here I'd characterize as the end of campaigns as we know them.

Or you could call it the radical transformation of campaigns from an analytic snapshot in time, a hectic ideation and creation process, and for the most part a linear execution of a set of creatives to achieve a particular marketing goal.

We're seeing something that's more of a 360degree engagement strategy with consumers and influencers on consumers.

An engagement strategy in this context is a multichannel online/offline strategy that focuses and begins to harness elements like social media, social networking and mobile and word-ofmouth networks, and also guerilla street teams and event sponsoring.

So the media mix has become much more diverse and much more dynamic. It's become more of a network running a circuit within a network, as opposed to a linear execution.

Have you seen this kind of change in terms of how entertainment and movie studios are beginning to think about their marketing? SB: Absolutely. I think this is so relevant, once again.

There are three important points to consider here. First, the lifecycle of content is vastly reduced; secondly, the consumer attention span to content is significantly low; and thirdly, there is an explosion of all sorts of content in the market. It is not all prime content, but it is relevant content and that's what matters today.

The only way a company can make its campaign effective is if they're present wherever the consumer is. We don't call it follow the consumer but rather preempting the consumer move. This is an attempt to assume a 360-degree presence with the consumer, and a lot of analytics are involved to make it 360. It demands a complex media mix.

Now if you are targeting social networking platforms and you know that you have covered the major ones, you will have covered a significant chunk of your target consumers. But then you also have a significant segment that is not into the Facebooks and the YouTubes, and as a marketer you can't forget about them overnight. Hence, traditional marketing continues as well. This is also one of the reasons why the cost of marketing has gone up in recent days.

MM: It would also seem that it focuses on what I'd call a "brand interaction strategy." Essentially, engagement with consumers transpires through a joint brand value-creation process, or a brand story-telling process. The brand interaction moves the consumer from awareness to consideration, trial, maybe even purchase and repeat purchase - and/or into advocacy.

The brand interactions in this model tend to evolve through a brand lifecycle. How have you seen entertainment studios begin to understand that the nature of content needs to change? SB: Historically, entertainment companies were never in direct contact with its consumers.

From that perspective, there is a sea of change in terms of strategy that the companies are adopting. Suddenly from a very passive kind of brand creation, which used to be the norm in earlier days, you find that an engaging, personal, direct, one-to-one kind of interaction has become the norm. This is all because of the web and mobile technology, and the way the technology has played out over a period of time.

I don't think that there's a choice for companies to go back to the older ways of doing things. Customers today would like to participate and engage - not be told. Just look 
at the success of the reality television shows and the entire euphoria about voting in these shows. With the web, mobile technology and social networks, it's all about engaging. That's the only way the customer would understand your brand's value proposition.

MM: One thing in particular that entertainment studios address is that when they launch a media property like a film or a major music star, the type of interactions that they produce are not just with tens or hundreds of thousands of people, but in many cases, millions or tens of millions of people.

For the most part, these millions or tens of millions of people essentially constitute unknown visitors to one of their various web properties.

Could you speak to the notion of the grand scale that entertainment firms have to work with? How do they begin to address the conversion of unknown visitors to known parties?

SB: I think there are two ways to look at it. One is probably looking at each individual customer and finding out who is reading what. The second is more of a profiling.

It may not be feasible to track each individual customer and know exactly what people are doing. But there is obviously the potential to track the profile of the customers who are reading/viewing the content.

From that perspective, if you launch particular content on your website or any other website and see the content flow through millions of consumers, there's the possibility of tracking the profile of the consumer from the website. Once you have the profile, you can then target a specific sect of consumers more effectively or design a campaign targeting them.

I won't go into direct marketing to an individual, as that borders on the privacy issue. I think profiling of customers would be a much better way to manage marketing initiatives.

MM: Doesn't that put an extraordinary level of stress or demand on the web services infrastructure? Are entertainment firms addressing this manifold demand for infrastructure services?

SB: I don't think that entertainment companies are keeping it all to themselves in terms of promoting the content. Some of the content you'll find on their websites. But people are not shying away from distributing content to popular platforms.

For example, entertainment companies are putting their own content on Facebook, or YouTube, which relieves a lot of the distribution headache in terms of where it goes. After that, all it involves is viral marketing, as the entertainment company is not responsible for maintaining the infrastructure.

While saying that, you obviously want to know what kind of profile is coming and consuming your content from your own website. So definitely, you need to upgrade your own website.

MM: So as we see the interactions with consumers increasing via social networking channels, this creates an avalanche of interaction data and traffic data. What have you seen in terms of developments and technical systems for really harnessing that new wealth of data about what's going on out in the market?

SB: I think this is probably more recent in terms of how people are using this data. We are seeing there is a significant increase of Business Intelligence (BI) infrastructure in companies. Many of these cases are just simple reporting of data, and not analytics.

Soon you may see high-end data-mining solutions coming in for entertainment companies in order to figure out the trends of media consumption. I have not seen this start in any big way yet, probably because many of these solutions are fairly new. But I think most of the companies are putting infrastructure in place for BI specifically for data-mining solutions.

MM: It seems to me that one of the challenges with many of the data mart and BI technology is they require an edit/transform/load of rows and rows of data into relational data stores. That's great for managing oceans of data, but horrible in terms of any kind of iterative predictive scenario building, when you're drilling down on various sets of data.

What have you seen in terms of developments in BI, and the role of special analytic databases as opposed to the standard BI corporate data mart?

SB: You're absolutely right. In terms of making a huge database and then only putting some query infrastructure in place, it's probably not going to work in a dynamic environment where 
you have to make a decision in a very, very short time.

We're finding in a lot of cases that people are building corporate dashboards. In a sense, you can have a snapshot of how things are working. If you see a trend in a particular way, then drill down and see where things are going. That's one of the trends happening.

The second thing is that instead of getting into mega databases encompassing every piece of consumer data, it is probably a better idea to do things in smaller data marts to investigate specific areas.

The only problem with this specific approach would be maintaining these kinds of individual data marts and the cost of maintaining that kind of infrastructure.

MM: Also, the time it would take to specify, build and launch a data mart would probably exceed the time that you had to execute whatever insight that analytic data mart would produce.

SB: Yes, time is an issue here. You probably can't build a data mart and execute on the basis of analytics of the data mart at the same time. To speed up the process, companies are already outsourcing some of the basic data-crunching jobs or what may be called pre-analytics and keeping the second level of analysis within themselves. That way, you don't have to wait for the entire analysis to be completed to figure out that something has grossly gone haywire. As far as data analytics infrastructure goes, it is kind of a mixed model now - some are run as SaaS and some in-house.

MM: In our tracking of the more advanced or leading-edge marketing analytic strategies, there's been another sea change or a radical shift in the overall approach to analytics. That is to say, we've seen that traditionally, marketing analytics tend to look back on historical data, and, using a variety of analytic techniques, try to forward predict and create forward-looking scenarios, as far as to what will happen. But it's all based on history.

It takes an exquisite amount of data cleansing. Most of the data systems producing the data never really anticipated this kind of analytic operation. I agree with you that there's some sort of pre-analytic - you can call it database hygiene - that can and should be outsourced. But a more fundamental thing we've seen has been a shift from the underlying assumption of analytics — which was basically statistics - to what I'm now seeing, which is econometrics.

As we start to look at the next wave of innovation - the next wave of breakthrough strategies in terms of marketing and marketing operations - there's a shift to what I'm now calling applied game theory in econometrics, and in using those particular disciplines by which to develop fact-based or analysis-driven engagement strategies that follow the customer through all the major cycles of brand interaction.

Have you seen anything that supports that particular way of looking at it?

SB: Personally speaking, I have not seen a game theory being applied to marketing innovations in the entertainment industry. But from a very conceptual point of view, you're right. You really don't have too much history to bank on. The way that things are changing, we just can't rely on historical data to predict customer behavior. We have seen application of such advance analytics in the retail sector and there is no reason why this should not be applicable here. MM: However, from what you know, does it logically extend to what would be a reasonable future scenario?

SB: I would imagine. It's also a matter of cost. Obviously, structuring any solution based on these kinds of advanced analytics is not going to be easy. It's not like you'll have resources to construct those readily available. But considering the fact that (if I take a rule of thumb), 20 per cent of the cost of a movie likely goes into marketing, and a significant chunk of that money goes to mobile, web and social networking platforms, you can imagine the kind of money that's going in that direction. Given that scenario, I think money should not be an issue with advanced analytics in the near future. I would bet that within a year or so it will become a large area of investment.

MM: We have seen the emergence of what is called a "Social Media Agency." The social media agencies create optimization strategies for viral marketing. In many cases, they are developing gaming interaction platforms, which they call "promotional platforms." They're immersive video games that have all of the same kinds of social dynamics represented in terms of incentives, challenges and rewards. 
I've seen the emergence of promotional platforms that have begun to incorporate everincreasing levels of game theory and, to a lesser extent, econometrics.

What I find interesting, though, is that at North American firms, the statistical and mathematical background that you need really has almost exceeded the output of our educational system here.

It seems to me logical that some of the more advanced analytic capacities would essentially migrate to those particular societies that have an education system producing hundreds of thousands of $\mathrm{PhDs}$ in mathematics and informatics and information technology.

Could you speak to what you see as the emerging role of outsourcing some of this BI and analytics to countries in areas such as India and China?

SB: I would say that for every resource that's being utilized by countries outside the US, you will always find a counterpart in the US. For knowledge process outsourcing, it's virtually impossible - from what I've seen - to do it in isolation. There's always a business context that is required to do these kinds of analytics. You cannot do it in isolation. It's a little different from software development. In the context of resource location, once again it will probably be a matter of supply vs demand. MM: Are you saying that in order to unlock the value of analytics and $\mathrm{BI}$, a business context is needed? And if we examine the business context, what you see is not only a set of mental maps and assumptions and social norms, but you also have in that business context a set of social interactions. Interactions between the analyst and a marketer and a content specialist that are essential to really kind of quickly turn raw data into insights, and insights into actions or strategies. SB: Absolutely. That engagement is absolutely essential in terms of unlocking this potential. That, many times, creates a challenge. Software development does not require that level of interaction. This is like you're really unlocking the business by analyzing data.

The kind of interaction here is far more involved. The speed at which this kind of knowledge can grow is probably limited by the availability of this kind of interaction.

MM: As we step back to recap our discussion on the marketing supply chain, you've indicated that first of all there are a number of changes that seem to converge.

One is that the marketing organization has begun to pull back in some of the value that ad agencies and service providers used to provide. In part, this reflects the broadening array of brand touchpoints - be they traditional offline touch-points or part of the burgeoning set of online touchpoints.

These online touchpoints have forced marketing organizations to rethink the whole notion of a campaign - thinking more in terms of a 360-degree engagement, and towards moving customers through a series of interactions of a brand lifecycle.

This also produces lots of data. New data that has challenged the traditional marketing analytic function and forces that marketing analytic function to move from a historical look back to something that's more real-time is immediately executable.

What else have you seen, in terms of the evolution of the marketing supply chain into what we might call more of a 100 per cent digital supply chain (Figure 1).

SB: From the point of view of the entertainment companies, we have observed a couple of general trends.

First, because of the collapsing of the content windows, the division-centric view of digital marketing platforms is getting blurred. As a result, there is a significant move towards the consolidation of all the digital platforms within a company.

Secondly, Digital Asset Management Systems (DAMs), which started off as archival systems to hold media content, have slowly but surely become a part of an overall supply chain. The discrete and isolated nature of these systems is being replaced by integrated workflows that surround these systems.

Thirdly, marketing content in the online space used to consist of small images or text. Now you have significantly larger content like longer video trailers or games that are part of an online campaign. This has created its own challenges in the digital media space.

Hence, on one side, the first two points are drivers for consolidation, while the third point is making the case for a distributed architecture. Although we must concede that in the age of digital cinema, the distribution of a $10 \mathrm{~min}$ 


\section{Source Content}
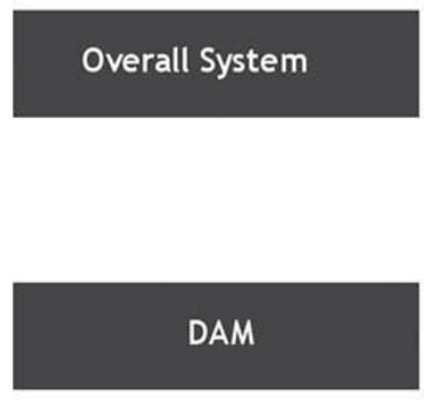

Marketing material destination
From

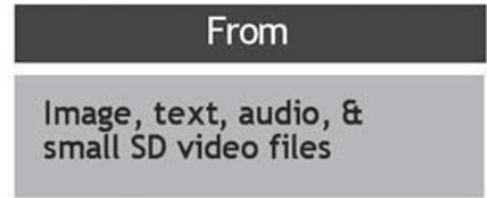

\section{Multiple \\ division/workgroup \\ specific digital \\ marketing ecosystem}

Stand alone DAM with

little or no integration

with upstream or

downstream systems in

the ecosystem

Print, TV and limited

web and mobile as

destination of content
To

Large SD as well as HD video files
One single consolidated system or multiple system connected through SOA framework

Fully integrated DAM, with upstream and downstream integration with other applications in the ecosystem
Mobile and Social networking sites are emerging as the key marketing platforms

Figure 1: Changes in the Digital Marketing Supply Chain

trailer is not a big issue. Nevertheless, the existing infrastructure is getting stretched. MM: As we move toward the end of this interview, are there any other kinds of thoughts - either forward looking thoughts and ideas or more of the practical considerations involved for marketing organizations making this shift?

SB: There are three things I'd like to say here. One is in the approach from the technological point of view. You need to re-evaluate your architectural strategy for the digital marketing supply chain in case it is not really forwardlooking or it is not able to support future growth. If that's the case, this could be the right time to re-architect it, so that the future growth is supported.

MM: This architecture would inherently be a service-oriented architecture with a notion of distributed web services, correct? Any other detail in terms of that marketing information architecture?

SB: Yes. Absolutely. I think you said it absolutely right. It has to be service-oriented. One thing you have to think about is that many companies today have multiple DAM systems - 15, 20 or even 30 systems. Consolidating all these systems into one large DAM may not be an optimum solution.

What would be more relevant is to keep these DAM systems intact, and probably bringing in the services on top of them as a common layer.

You have the systems - storehouses - on the bottom. Those are just working as an archival system. On the top of that, you have all the workflows and all the services on a services layer. I think that's the way the trend is happening, from a technical point of view (Figure 2).

SB: The other point I'd like to mention is on the mobile side. I think there's a huge transformation happening. In terms of requirements, it's exploding. Going forward, we can see mobile marketing getting bigger and bigger.

Given the number of carriers and handsets and the lack of standards and other challenges, we might see the software as service model becoming more prominent in this particular area. That way, most of the marketing companies can keep their costs at a minimum, 

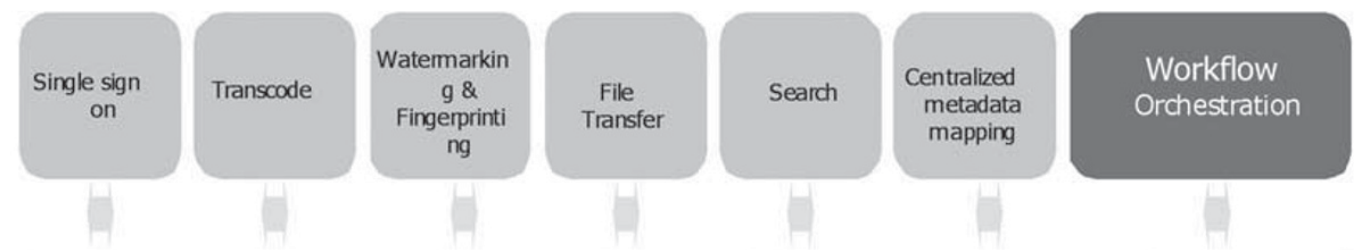

SOA -Enterprise Bus
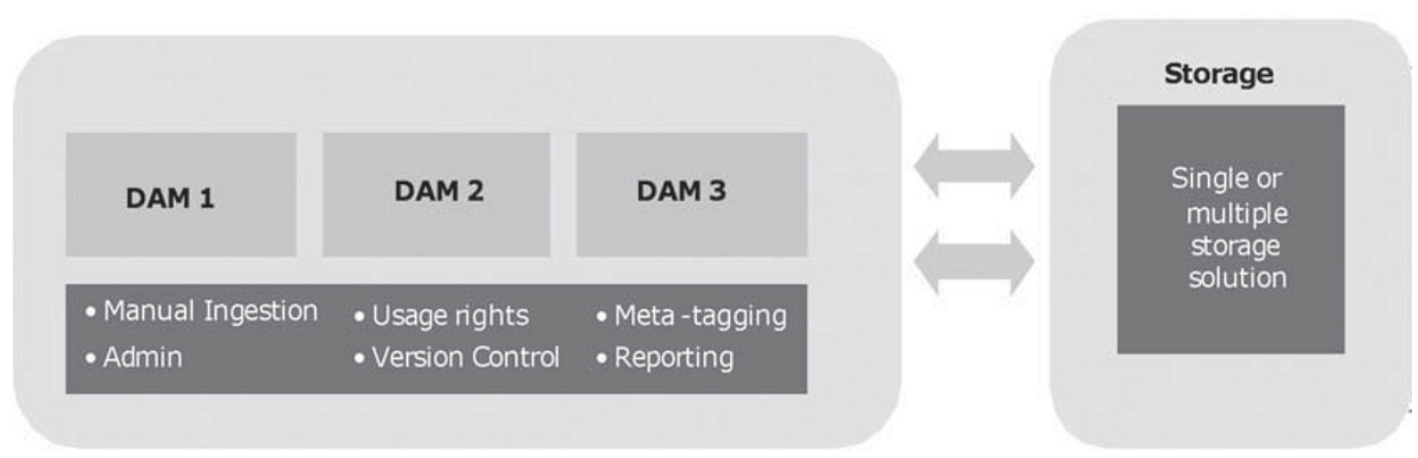

Figure 2: Simplified HCL's "IMPACT" framework for SOA-based digital marketing supply chain

and use the services of the service provider in taking the content and reaching their destination.

That is one of the trends that I'd say you'll see in the next couple of years' time. That's an area in which marketing companies may not invest. There are many companies in the world that specialize in this specific area, and probably would be better off providing that as a software service.

MM:Yes. I agree completely.

The third thing is obviously on the social networking side.

MM: One last thing on SaaS - doesn't that put a greater and greater emphasis on how you integrate multiple services — or I should say services from multiple providers? And how do you establish effective governance both in terms of policy and day-to-day operation of all of these services?

SB: I think it's much easier than how it might sound as a complex process. At the end of it, you're not really interacting with a lot of companies. In some sense, it actually simplifies the operation.

If you look at it today, what does a media company do if you have to distribute mobile content? It's actually taking services from a thirdparty vendor to transcode content. Then, once again, they are utilizing a third party to put it in the carrier's environment. And at the end they are taking all the intelligence/data back from the carrier for interpreting the effectiveness of the campaign. In this process, you have to constantly worry about new operating systems, new formats laid out by carriers and new handsets.

All of this is kind of discrete operations. In a sense, if you get rid of all of these back and forth operations, concentrate your efforts on making the best marketing campaign that you can create, and then utilize one SaaS provider to handle the operations side while helping you implement all your strategies one after another without necessarily burdening you with the technical aspects of the process, that would be a great value add.

MM: Excellent.

You had a third point?

SB: The third point is obviously on the social networking platform. This is one area in which once again it can be software as a service, or an ASP model. In a sense, there is no limit to social networking and what we really want to build. 
If you look at any social networking platform today, all they're providing you with is the open APIs. People are putting all the hooks in and coming up with lots of different applications.

That's probably the way to go into it. Social networking platforms are all a matter of how creative you are in terms of giving that interactive platform to the consumer.

Investing millions of dollars on that may not be a very good idea. You'd probably be better off having an aggregator develop and build that platform. Individual companies can actually leverage that platform. That way the cost is going to be shared, risk is going to be shared and it's much more economical in all aspects.

MM: Excellent. Again, thank you very much.

SB: Thanks for this call. It was a pleasure talking to you. 